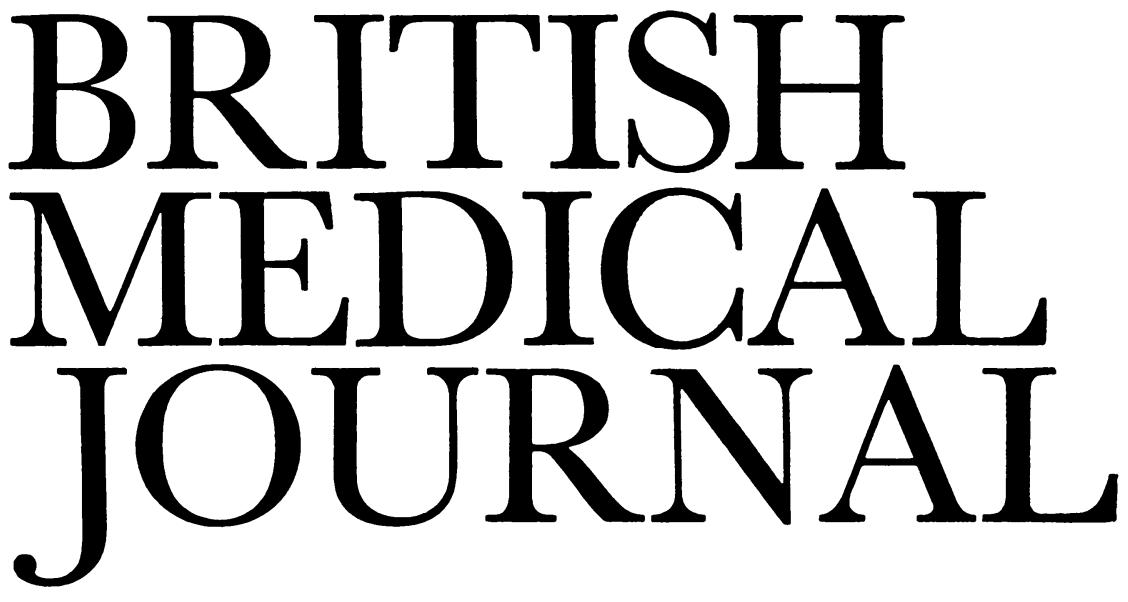

\title{
Follow up of patients with breast cancer
}

Questions are often asked about both the value and the appropriateness for many patients of hospital follow up. "Most general practitioners are aware," said a letter to the $B M \mathcal{A}$, "that their patients with breast cancer are often unhappy with their routine consultations in the surgical outpatient department." The reasons cited included overcrowded waiting rooms, cursory consultation by junior staff, lack of continuity of care, and conflicting advice on consecutive visits. Is there, indeed, any advantage from regular follow up in hospital compared with leaving the patient and her general practitioner to detect recurrence and take appropriate action? Clearly we should first ask "Why?" before trying to determine a policy which will reflect who, when, and where.

One reason for continued follow up is research or accumulation of data. In a specialist unit or one taking part in a multicentre trial accurate information will need to be gathered-but that will require experienced, dedicated, and committed doctors. The clinical findings of short term registrars of varying experience are unlikely to be reliable. Follow up may also be justified to audit an individual unit, but only if the clinician regards local or regional recurrence as an indictment of his own judgment and technique. There can be little benefit if local or regional recurrence is accepted as inevitable and a trivial matter.

A third justification for hospital follow up is the detection of recurrence, either local or systemic, as early as possible. Many studies have shown that routine follow up for systemic disease-for example, by bone scans to detect bone metastases-is not cost effective. ${ }^{2}$ The picture is less clear for local and regional recurrences, where the balance differs with circumstances. When the primary local treatment is radical, local recurrence occurs mainly in the first two years and is usually accompanied by systemic recurrence. ${ }^{3}$ In these patients early detection and treatment has no clear advantage over allowing them to detect recurrence themselves. The same may not be true for patients managed by conservative local treatment. Simple mastectomy alone carries a local and regional recurrence rate of at least $25 \%$ (compared with less than $10 \%$ for formal Patey mastectomies), and many of these patients will not develop systemic recurrence for a long period-suggesting that local treatment of early recurrence may be of value in salvaging some at least. With lumpectomy and radiotherapy local and regional recurrences are split evenly around five years and appear for at least 15 years, possibly indefinitely. ${ }^{4}$ Early salvage mastectomy may improve survival-a good reason for continuing careful follow up. ${ }^{4}$ The more conservative the local treatment the more likely that some of the increased local recurrence will occur without systemic disease and warrant aggressive local treatment with some hope of cure.

A fourth reason for follow up is to detect cancer of the contralateral breast at an early stage. In a recent paper from the Guy's Hospital group cancer in the opposite breast developed at a constant rate and showed no tendency to increase or decrease with follow up..$^{5}$ It correlated with age, however, because women who developed their first breast cancer under the age of 40 had three times the risk of developing a second breast cancer than did those who developed their first cancer after the age of 40 . Overall this risk of a metachronous contralateral cancer was 5.9 times that of the risk in the first breast in the general population. Many studies have shown that a contralateral breast cancer carries no worse prognosis than that of the first breast, so early detection and treatment is clearly worth while. ${ }^{67}$ Hard data on the long term survival of patients with breast cancer, such as those provided by Duncan and Kerr, provide overwhelming evidence that tumours detected at a small size carry a definite survival advantage, which persists to 20 years. ${ }^{8}$ Hence there is a sound case for regular follow up to detect contralateral cancer at an early stage, particularly in those with a long survival expectancy-such as younger women with tumours of good prognosis. The evidence reported by Johnston-suggested an increased rate of contralateral cancer among patients having adjuvant radiotherapy compared with those having radical surgery alone-points to another group requiring careful long term follow up. ${ }^{9}$

We apologise to readers for the late arrival of their journals. This is being caused by industrial action at the printers.

Readers are reminded that the $B M$ F's current classified job advertisements are also available on Prestel. 
Careful follow up for local and regional recurrence is, then, worth while and indeed mandatory in patients treated by conservative measures, since the high local recurrence rate associated with these procedures may still be amenable to radical local treatment. Local recurrence in an irradiated breast and axillary recurrence after mastectomy may be difficult to detect at an early stage and can progress to extensive disease within a year, so follow up by an experienced consultant at perhaps four monthly intervals is indicated-and must continue indefinitely, putting a heavy load at senior level on to the hospital clinic. There is little reason for follow up to detect distant metastases or local or regional recurrence in patients who have been treated by radical local measures, though a note of caution is required in using a term such as radical surgery. The $7-8 \%$ local recurrence which should be achieved with Patey or Halsted mastectomy requires conviction and close attention to technique. These operations have been associated with 10 year local recurrence rates ranging from $6.3 \%$ for all stages I and II cases ${ }^{10}$ to $36 \%$ for stage II cases. ${ }^{11}$

The balance is finally tipped, however, by the strong case for following up all patients to detect contralateral cancer. Clearly any policy will depend on the patients, their initial treatment, and circumstances, but we believe follow up in hospital seems inevitable. Patients who have had careful "radical" local treatment (such as radical mastectomy, formal Patey mastectomy, or simple mastectomy and radical radiotherapy) need only a "screening" type of follow up to detect contralateral cancer. The interval between breast screening is controversial, but our policy is to see patients annually for physical breast examination with mammography of the contralateral breast every two years. Patients are taught self examination and are advised to report any intercurrent abnormality to their general practitioner. Local recurrence after mastectomy is usually recurrence in the flap and is easily detected by the patient. The general practitioner is able to arrange an early appointment in the breast clinic if he thinks it necessary.

Our hope in the long term is that effective systemic treatment will make the problem of local recurrence an historic one, but from our current perspective this still looks a very distant prospect.

L E HUGHes

Professor of surgery

S P COURTNEY

Research fellow

Department of Surgery,

University of Wales College of Medicine,

Cardiff CF4 4XN

1 Clark PB, Morris DL. Management of patients after mastectomy. Br Med f 1981;282:2095-6. 2 Turner DTL, Ackery D, Fraser JD, Chant ADB. The bone scan-has annual screening a place in the follow up of breast cancer patients? Clin Oncol 1977;3:126-7.

3 Donegan WL. Local and regional recurrence. In: Donegan WL, Spratt JS, eds. Cancer of the breast. Major problems in clinical surgery V. Philadelphia: W B Saunders, 1979:484-503.

4 Osborne MP, Ormiston N, Harmer CL, McKinna JA, Baker J, Greening WP. Breast conservation in the treatment of early breast cancer. Cancer 1984;53:349-55.

5 Chaudary MA, Mills RR, Hoskins EOL, et al. Bilateral primary breast cancer: a prospective study of disease incidence. Br $\mathcal{F}$ Surg 1984;71:711-4.

6 Khafagy MM, Schottenfeld D, Robbins GF. Prognosis of the second breast cancer. The role of previous exposure to the first primary. Cancer 1975;35:596-9.

7 Haagensen CD, Bodian C, Haagensen DE. Breast carcinoma risk and detection. Philadelphia: W B Saunders, 1981.

8 Duncan W, Kerr GR. The curability of breast cancer. Br Med $\mathcal{F} 1976$;ii:781-3.

9 Johnston FRC. Adverse effect of postoperative irradiation on the breast. Advances in Medical and Oncological Research Education 1978;12:401.

10 Haagensen CD, Bodian C. A personal experience with Halsted's radical mastectomy. Ann Surg 1984;199:143-50. 11 Lythgoe JP, Palmer MK. Manchester regional breast study-5 and 10 year results. Br f Surg
1982;69:693-6.

\section{Coagulase negative staphylococcal infections}

Staphylococcus epidermidis-or the coagulase negative staphylococcus as it should more accurately be called-is being recognised increasingly often as a pathogen. Deep seated infections with coagulase negative staphylococci may present months or even years after the insertion of prosthetic heart valves ${ }^{1}$ or in patients given artificial hips ${ }^{2}$ and these are the most common organism causing infection in patients with Spitz-Holter valves. ${ }^{3}$ Infections with coagulase negative staphylococci have also been described in patients undergoing continuous ambulatory peritoneal dialysis, ${ }^{4}$ in some but not all neonatal units, ${ }^{56}$ and in patients with leukaemia. ${ }^{7}$ In a series of 182 episodes of catheter associated bacteraemia at St Thomas's Hospital coagulase negative staphylococci were responsible for over one fifth of infections. ${ }^{8}$ Staph saprophyticus is recognised to be a common cause of urinary infections in young women and will not be discussed here.

Despite the increased recognition of these organisms as pathogens both diagnosis and the treatment of infection remain difficult. A single blood culture isolate of a coagulase negative staphylococcus may be dismissed as a contaminant, and repeat cultures must be obtained if the patient has a prosthesis in situ or falls into one of the other risk groups. Repeated isolation of coagulase negative staphylococci from blood cutures is usually taken as evidence of infection, although comparison of the antibiotic sensitivities and (if possible) the biotypes of the isolates will help to confirm that the same organism is being identified. A recent American study took this a stage further by analysing the plasmid content of isolates of coagulase negative staphylococci from the same patient and found that this technique helped in differentiating between pathogenic and contaminant strains. ${ }^{9}$ This approach would be unsuitable for routine use in ordinary microbiology laboratories.

A system of classification of the different species forming coagulase negative staphylococci was put forward by BairdParker $^{10}$ and by Kloos and Schleiffer," but again the technique is often too time consuming for routine identification of isolates. A more rapid technique has been introduced, ${ }^{12}$ but its results do not always correlate with those of Kloos and Schleiffer or Baird-Parker. A system of reverse phage typing is also available in certain cases.

Sensitivity tests should always be done on individual isolates of coagulase negative staphylococci since their sensitivity pattern is much less predictable than that of Staph aureus. Coagulase negative staphylococci resistant to a wide range of antibiotics have been reported. ${ }^{15713}$ Richardson and Marples looked at 500 isolates over a three year period and found that $30 \%$ were resistant to gentamicin and $38 \%$ to methicillin. ${ }^{14}$ These findings have been confirmed in Britain ${ }^{5}$ and abroad. ${ }^{7}$ Transfer of resistance from coagulase negative staphylococci to Staph aureus seems rare, though it has been shown both in laboratory tests and in vivo. ${ }^{15}$ Not all units report multiresistant coagulase negative staphylococci, so microbiologists need to know the local sensitivity patterns of coagulase negative staphylococci -which may vary even within different units of the same hospital, as they do for Gram negative organisms.

The treatment of deep seated prosthetic infections due to coagulase negative staphylococci may usually be guided by results of sensitivity testing, but other infections (such as 